\title{
Statically Recrystallized Microstructure and Texture of Heterogeneous Nanostructured SUS316LN Austenite Stainless Steels
}

\author{
Hiromi Miura ${ }^{1, *}$, Masakazu Kobayashi ${ }^{1}$, Tomoki Tsuji ${ }^{1}$, Takahiro Osuki ${ }^{2}$, Takuya Hara $^{2}$ and \\ Naoki Yoshinaga ${ }^{3}$ \\ ${ }^{1}$ Department of Mechanical Engineering, Toyohashi University of Technology, Toyohashi 441-8580, Japan \\ ${ }^{2}$ Research \& Development, Nippon Steel Corporation, Amagasaki 660-0891, Japan \\ ${ }^{3}$ Nippon Steel Corporation, Futtsu 293-8511, Japan
}

\begin{abstract}
90\% cold-rolled SUS316LN austenite stainless steels with different Mn content were annealed and the changes in the microstructure and texture after static recrystallization (SRX) were investigated. A typical heterogeneous nanostructure (HN), in which "eye"-shaped twin domains, shear bands and low-angle lamellae were main structural components, was developed by the heavy cold rolling. The formation of twin domains with crystallographical orientation of $\{111\} / /$ rolling plane effectively suppressed a sharp $\{101\}$ texture evolution. However, preferential SRX nucleation at shear bands close to $\{101\}$ orientation hindered the above $\{111\}$ texture component and, then, a typical $\{101\}$ one prevailed in whole area after full SRX. Dense nuclei in the HN led to homogeneous evolution of fine-grained structure. While the effects of Mn content on the microstructure and texture were not evident, superior mechanical properties appeared to be more emphasized with increasing Mn content. [doi:10.2320/matertrans.MT-M2021231]
\end{abstract}

(Received November 24, 2021; Accepted December 3, 2021; Published January 17, 2022)

Keywords: SUS316LN austenite stainless steel, heterogeneous nanostructure, static recrystallization, texture, mechanical property

\section{Introduction}

It is well known that texture of steel sheets sensitively influences plastic deformability. For example, deep drawability of austenitic stainless steel sheets is drastically improved by $\{111\}$ texture on the rolling plane. ${ }^{1)}$ However, the texture control appears quite difficult because of the sharp $\{101\}$ texture development by heavy rolling and it hardly changes even after static recrystallization $(\mathrm{SRX}){ }^{2}{ }^{2}$ Various attempts have been carried out to modify the texture and one of the most effective methods should be employment of shear bands and mechanical twins induced by the different peripheral speed rolling. Inoue et al. have successfully developed $\{111\}$ texture in a SUS316L stainless steel by the warm rolling followed by SRX. ${ }^{3)}$ They explained this phenomenon by the formation of twins which $\{111\}$ planes became parallel to the rolling plane. This method employing the different peripheral speed rolling should be, however, practically difficult for the mass production in the factories. On the other hand, recently, Miura et al. have found the characteristic microstructure in which "eye"-shaped twin domains to align their twinning planes parallel to the rolling plane of SUS316LN stainless steel (SUS316LN). ${ }^{4)}$ Donadille et al. also indicated brass-type texture evolution in the heavily cold-rolled SUS316L stainless steel containing dense mechanical twins. 5) Therefore, their reports may bring a hope to suppress the evolution of sharp $\{101\}$ texture by the introduction of the "deformation-induced microstructures".

The texture after SRX is strongly affected by the initial one before annealing. ${ }^{1,2,5)}$ Nevertheless, it appears to be possibly controlled by utilizing deformation-induced microstructures such as shear bands, ${ }^{2,6)}$ mechanical twins ${ }^{5)}$ and so on, as mentioned above. This is because these can be the most preferential nucleation sites due to the higher dislocation density, larger strain gradient and much finer grain size. The

*Corresponding author, E-mail: miura@me.tut.ac.jp heterogeneous-nanostructured (HNed) SUS316LN is composed of twin domains surrounded by shear bands and they are further embedded in low-angle lamellae. ${ }^{7)}$ All the component microstructures are of nanometer size. The thermal stability of such ultrafine-grained structure is quite low and, therefore, SRX onsets at relatively lower temperatures than those of the metallic materials with normal grain sizes. ${ }^{8)}$ Furthermore, in case of the HNed stainless steels, a truly homogeneous nucleation could lead to the evolution of thermally stable equi-axed fine grains between 1 and $2 \mu \mathrm{m} .{ }^{9)}$ Grain refinement is believed to effectively improve plastic deformability as well as strengthening according to Hall-Petch relation.

In the present study, heavily cold-rolled SUS316LN to have $\mathrm{HN}$ was followed by SRX at relatively low temperatures to develop fine-grained homogeneous microstructure. And the evolved microstructures and texture were precisely examined by means of orientation imaging microscopy (OIM).

\section{Experimental}

$90 \%$ cold-rolled sheets with a thickness of $0.5 \mathrm{~mm}$ of various SUS316LN with different Mn concentration were prepared (Table 1). The different addition of $\mathrm{Mn}$ was to expect change in the stacking fault energy (SFE), which affects much to the probability of mechanical twinning. The critical shear stress to trigger dislocation motion for twinning is described as follows, ${ }^{10}$ )

$$
\tau=\gamma_{S F} / 2 b_{T}
$$

where $\gamma_{S F}$ is the value of SFE and $b_{T}$ is the Burgers vector of a twinning partial dislocation. Therefore, twinning becomes easier as SFE decreases. The value of SFE was evaluated using the equation proposed by Schramm and Reed. ${ }^{11)}$ While the difference among the modified SUS316LN was only about $21 \mathrm{~mJ} \mathrm{~m}^{-2}$, the value of $\tau$ would be $30 \%$ reduced. 
Table 1 Chemical compositions in mass \% of the modified SUS316LN stainless steels with different Mn content. Calculated stacking fault energy (SFE) using the equation by Schramm and Reed ${ }^{11)}$ is also shown. The hatched one is a typical chemical composition of the SUS316LN stainless steel.

\begin{tabular}{|c|c|c|c|c|c|c|c|c|c|c|c|}
\hline Sample name & $\mathrm{C}$ & $\mathrm{Si}$ & $\mathrm{Mn}$ & $\mathrm{P}$ & $\mathrm{S}$ & $\mathrm{Ni}$ & $\mathrm{Cr}$ & $\mathrm{Mo}$ & $\mathrm{N}$ & $\mathrm{Fe}$ & $\mathrm{SFE} / \mathrm{mJm}^{-2}$ \\
\hline $0.02 \mathrm{Mn}$ & 0.020 & 0.52 & 0.02 & 0.015 & 0.001 & 10.91 & 17.59 & 2.49 & 0.144 & Bal. & 50.2 \\
\hline $1.53 \mathrm{Mn}$ & 0.019 & 0.53 & 1.53 & 0.014 & 0.001 & 11.05 & 17.81 & 2.52 & 0.143 & Bal. & 56.3 \\
\hline $2.97 \mathrm{Mn}$ & 0.020 & 0.53 & 2.97 & 0.016 & 0.001 & 11.11 & 17.85 & 2.45 & 0.166 & Bal. & 60.7 \\
\hline $5.96 \mathrm{Mn}$ & 0.020 & 0.52 & 5.96 & 0.016 & 0.001 & 11.15 & 17.89 & 2.53 & 0.190 & Bal. & 71.3 \\
\hline
\end{tabular}

The SUS316LN sheets were annealed at various temperatures between $923 \mathrm{~K}$ and $1173 \mathrm{~K}$ in a salt bath for $1 \mathrm{~h}$ followed by water quenching and the evolved microstructure was examined using OIM after mechanical and electritical polishing. Microstructural observation was carried out at around the center in thickness and from transvers direction (T.D.) for the as-rolled samples and from normal direction (N.D.) for the annealed ones.

The annealed samples were tensile tested parallel to rolling direction (R.D.) on an Instron-type mechanical testing machine at an initial strain rate of $2.5 \times 10^{-3} \mathrm{~s}^{-1}$. The gauge dimensions of the samples were $6.0 \times 2.0 \times 0.5 \mathrm{~mm}^{3}$. Change in hardness after annealing was measured using micro-Vickers hardness tester.

\section{Results and Discussion}

A typical initial microstructure before annealing (i.e., as cold rolled) is displayed in Fig. 1. No specific difference among the evolved microstructures depending on $\mathrm{Mn}$ concentration could be found by OIM analysis. Even while shear bands around the twin domains were not clearly detected by OIM due to the high strain energy, ${ }^{5)}$ a specific feature of the HN, i.e., "eye"-shaped twin domains surrounded by shear bands and they were embedded in the low-angle lamellae, ${ }^{7)}$ is evidently confirmed. It is also
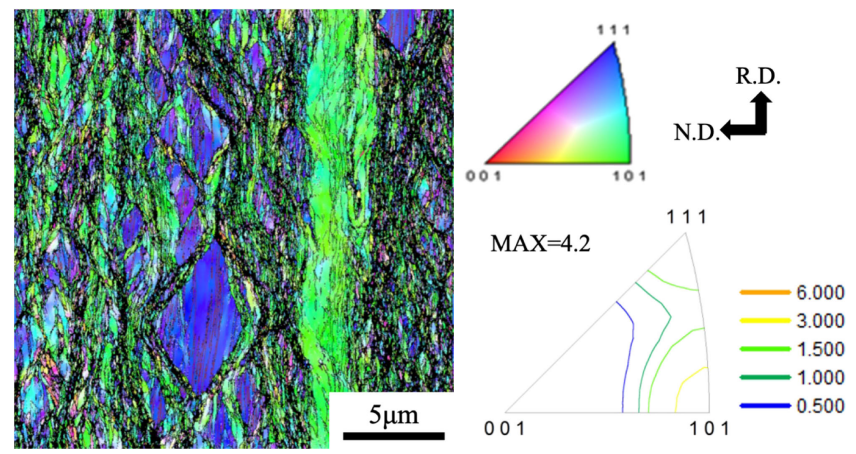

Fig. 1 Evolved heterogeneous nanostructure in the $90 \%$ cold-rolled SUS316LN stainless steel containing $2.97 \%$ Mn. N.D. and R.D. indicate normal and rolling directions. While observation was carried out along transvers direction, color mapping was decoded along normal direction for easier identification of the specific crystallographical orientation of the "eye"-shaped twin domains. The corresponding inverse pole figure with the maximum intensity are also shown. characteristic to see that the twin domains possess crystallographical orientation of $\{111\}$ twinning plane // rolling plane. Watanabe et al. further precisely examined the crystallographical orientation of the twin domains and revealed as $\langle 211\rangle / /$ R.D. and $\langle 110\rangle / /$ T.D., or $\langle 110\rangle / /$ R.D. and $\langle 211\rangle / /$ T.D. ${ }^{12)}$ These twin domains with the orientation $\{111\} / /$ rolling plane suppress the sharp $\{101\}$ texture evolution, while $\{101\}$ component owing to lowangle lamellae still retains.

The change in the hardness after annealing at various temperatures for $1 \mathrm{~h}$ is summarized in Fig. 2. The initial hardness of all the samples were identical independent of chemical composition. After showing slight hardening, the hardness rapidly dropped with increasing annealing temperature. As will be shown later, this softening is due to extensive occurrence of SRX. However, in the samples containing higher amount $\mathrm{Mn}$, the softening tended to be slightly delayed and the hardness after full SRX appeared higher. These differences would be derived by the solute drag effect and solid solution hardening by $\mathrm{Mn}$ and N. Anyway, full SRX could be achieved at around $1100 \mathrm{~K}$ irrespective of samples. The hardening of the HNed SUS316LN during annealing at around $800 \mathrm{~K}$ was also reported by Miura et al., ${ }^{7)}$ while the mechanism has not been defined yet.

The evolved microstructure after annealing at various temperatures for $1 \mathrm{~h}$ is exhibited in Fig. 3. The samples with

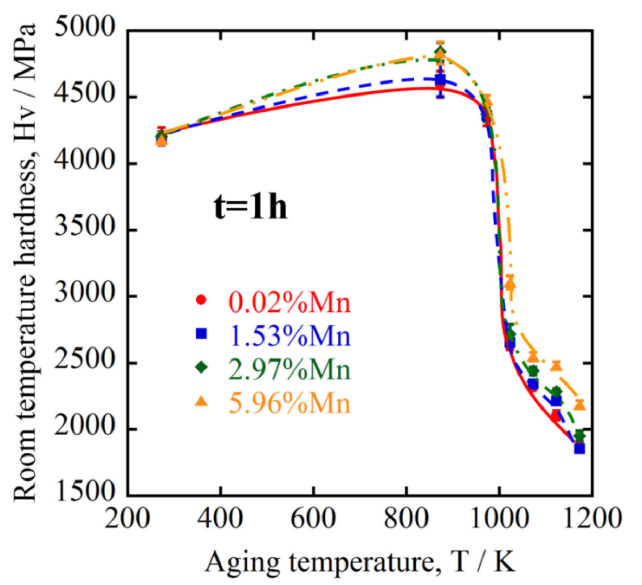

Fig. 2 Softening behavior by annealing at various temperatures for $1 \mathrm{~h}$ of the heterogeneous nanostructured SUS316LN stainless steels containing different amount of Mn. 


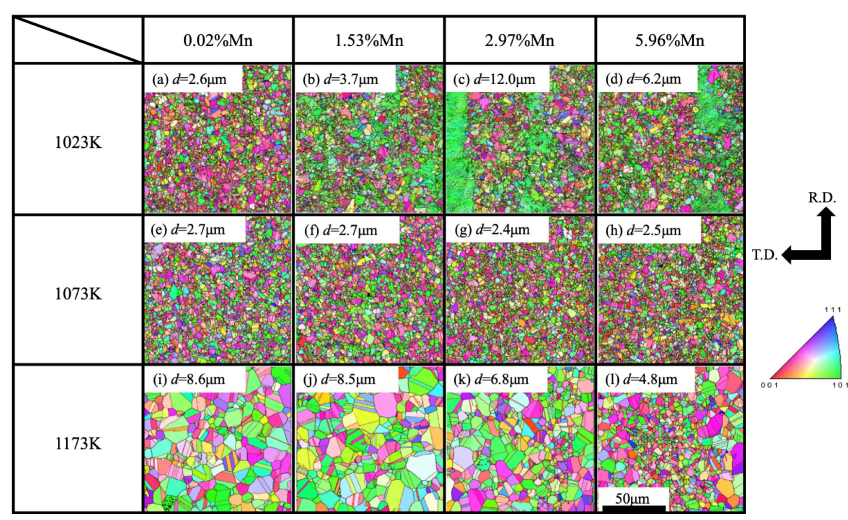

Fig. 3 Evolved microstructures after annealing at various temperatures for $1 \mathrm{~h}$ of the $90 \%$ cold-rolled SUS316LN stainless steels containing various amount of Mn. Observation was carried out from normal direction and color decoding is parallel to normal direction. The average grain size, $d$, is also shown.
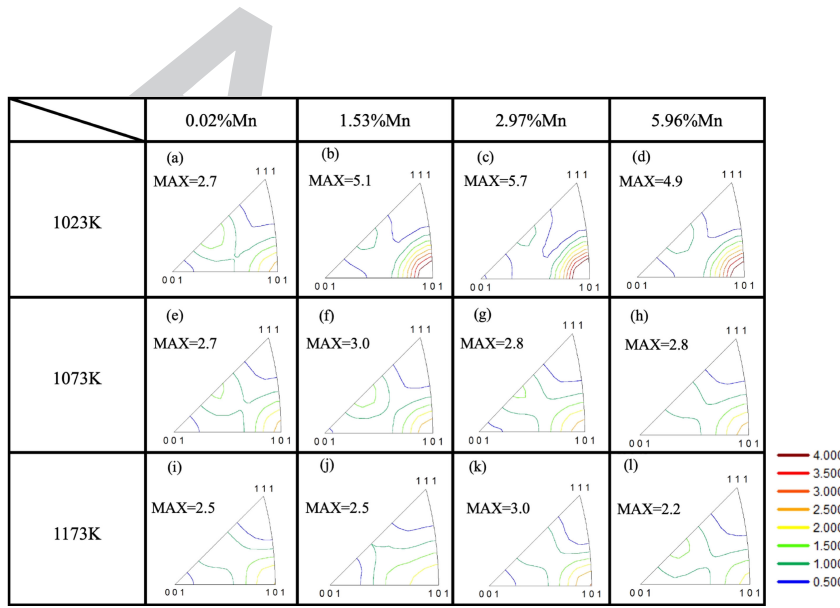

Fig. 4 Change in the texture after annealing at various temperatures for $1 \mathrm{~h}$ of the $90 \%$ rolled SUS316LN stainless steels containing various amount of Mn. The maximum intensity of each map is shown.

containing $\mathrm{Mn}$ more than $1.5 \%$ were not fully recrystallized at $1023 \mathrm{~K}$ and, therefore, elongated as-rolled grains with sharp $\langle 101\rangle$ texture can be observable. This result indicates that shear bands and/or twin domains are rather thermally unstable compared with low-angle lamellae and, hence, preferentially recrystallized. In contrast, the $0.02 \mathrm{Mn}$ sample was fully SRXed. When annealing temperature was raised to $1073 \mathrm{~K}$, homogeneous fine-grained structures with average grain size less than $3 \mu \mathrm{m}$ were attained. The average grain size increased with increasing annealing temperature and coarser grain size over $5 \mu \mathrm{m}$ in average was developed at $1173 \mathrm{~K}$. This grain coarsening was induced by secondary SRX, which is consistent with the result of slower softening over $1123 \mathrm{~K}$ in Fig. 2. The homogeneous fine-grained microstructure should be caused by high density of SRX nuclei due to high dislocation density, high strain energy in addition to ultrafine-grained structure of the HN. ${ }^{9}$ )

Changes in the texture evolved on the rolling plane are summarized by inverse pole figures in Fig. 4. It is evident that the texture was drastically changed by SRX from the initial one in Fig. 1. That is, $\{111\}$ component has almost disappeared and, instead, $\{101\}$ one evidently increased. And also $\{112\}$ one slightly appeared. Therefore, the initial

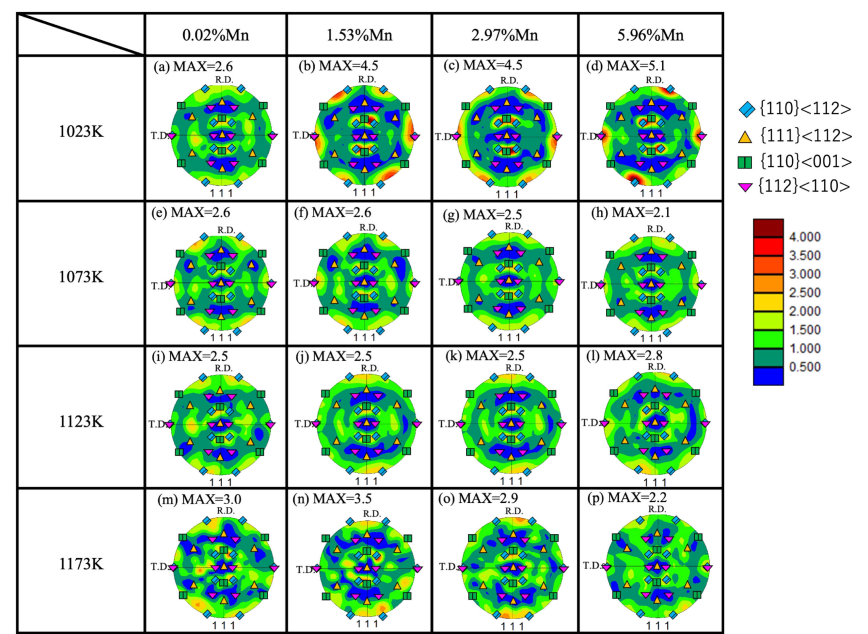

Fig. 5 Change in the texture expressing by pole figures after annealing at various temperatures for $1 \mathrm{~h}$ of the $90 \%$ rolled SUS316LN stainless steels containing various amount of Mn. Observation was carried out from normal direction and color decoding is parallel to normal direction. T.D. and R.D. are the transvers and rolling directions. Symbols indicate major texture components.

texture was almost completely replaced to new one after full SRX irrespective of the amount of Mn content. The texture attained was not largely different from those commonly observed in the SRXed austenitic stainless steel sheets. ${ }^{1-3)}$ It is also interesting to see Fig. 4 that the intensity of the \{101\} component tended to gradually decrease with increasing temperature. This should be presumably due to multiple twinning during secondary SRX to weaken sharp $\{101\}$ texture.

Figure 5 is the replot of the evolved texture shown in pole figures. It is confirmed in Fig. 5 that $\{101\}$ components induced by cold rolling (Figs. 5(c) and (d)) were much lowered by the occurrence of SRX. No substantial changes depending on $\mathrm{Mn}$ content nor annealing temperature could be observed as was in Fig. 4. However, the evolution of Goss component as well as brass one before and after SRX is apparent from Fig. 5, while the intensities are not so high. This result should suggest that these texture components were originated from SRX nucleation at shear bands, as will be discussed below.

When the most preferential nucleation takes place at twin domain, rather sharp $\{111\}$ component shown in Fig. 1 should never disappear because of sequential multiple twinning accompanied by grain-boundary migration invading neighboring grains. Nevertheless, the $\{111\}$ component appeared quite minor one already even at the beginning of SRX (see Figs. 3(b), (c), (d)). On the contrary, new SRX grains with orientations with N.D. // $\langle 110\rangle$ and $\langle 112\rangle$ look dominant. This argument clearly denies the most preferential nucleation at "eye"-shaped twin domains. Koga et al. have precisely investigated mechanical properties and crystallographical orientation of the microstructural components in the HNed SUS316LN. ${ }^{13)}$ They reported that extremely large shear strain higher than 6 was applied at shear band and, hence, high angle boundaries with misorientation angle over 20 degrees in average were evolved. The high-angle grain boundary, namely high energy one, is known to easily 


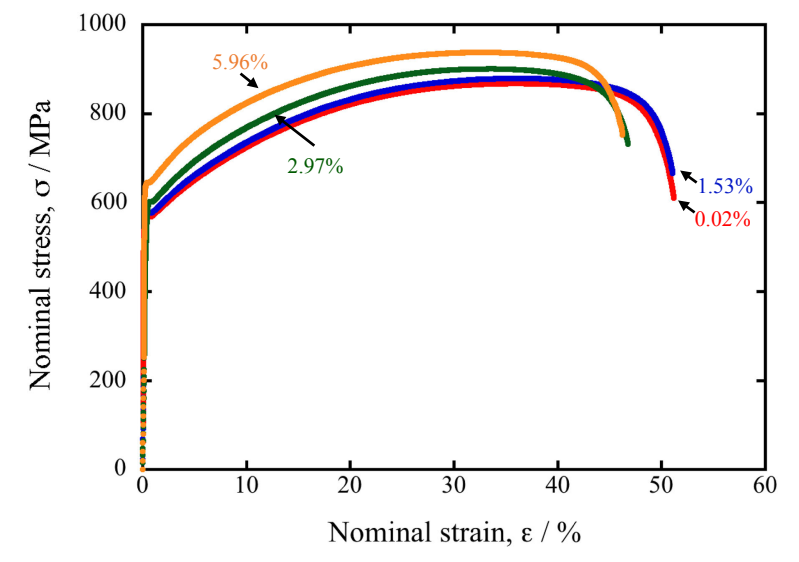

Fig. 6 Tensile properties of the fully recrystallized heterogeneous nanostructured SUS316LN stainless steels after annealing at $1073 \mathrm{~K}$ for $1 \mathrm{~h}$ The amount of added Mn in mass $\%$ in each modified SUS316LN stainless steel is shown.

migrate to form new grains behind the migrating boundary. ${ }^{14)}$ They have also shown that these shear bands possess orientation distribution close to $\{101\}$. In contrast, the twins and low-angle lamellae, i.e., low energy boundaries, are known to be thermally stable. ${ }^{14)}$ In short, the preferentially nucleated SRX grains having orientation close to $\{101\}$ at shear bands grew and invaded the neighboring areas to develop $\{101\}$ "mild" texture. This analysis is consistent with the report by Donadille et al..$^{5)}$ that preferential nucleation at shear bands and a texture evolution close to $\{101\}$ occurred in a cold rolled SUS316L stainless steel. Koga et al. have revealed rather large misorientation distribution among the component grains in the shear bands. ${ }^{13)}$ It is assumed, therefore, that the appeared $\langle 112\rangle / /$ ND component after SRX would be affected by the orientation distribution in the shear bands.

As a brief summary, although sharp $\{111\}$ component was successfully developed in a heavily cold-rolled SUS316LN by the evolution of $\mathrm{HN}$, it was almost vanished by preferential SRX nucleation at shear band having texture close to $\{101\}$ to hinder $\{111\}$ component. Therefore, suppression of preferential SRX nucleation at shear bands is necessary to retain $\{111\}$ texture component even after annealing of HNed SUS316LN.

Tensile properties were investigated and the flow curves are displayed in Fig. 6. The yield strength and ultimate tensile strength (UTS) increased gradually but slightly with increasing Mn content. It is notable to see that yield point phenomenon took place clearly in all the samples. The yield strength and UTS are $561 \mathrm{MPa}$ and $867 \mathrm{MPa}$ for $0.02 \mathrm{Mn}$ sample, whereas $642 \mathrm{MPa}$ and $940 \mathrm{MPa}$ for $5.96 \mathrm{Mn}$ one, respectively. Relatively large uniform elongation could induce good ductility of $51 \%$ and $46 \%$ as well. A good mechanical property was, therefore, attained by SRX of the HNed SUS316LN. These well-balanced mechanical properties should be, however, mainly caused by the homogeneous grain refinement.

\section{Conclusions}

A sharp $\{111\}$ texture component was successfully developed on the rolling plane by heavy cold rolling of SUS316LN austenite stainless steels (SUS316LN) due to evolution of "eye"-shaped twin domains, which is one of the dominant component structures of the heterogeneous nanostructure $(\mathrm{HN})$. Nevertheless, the $\{111\}$ component was vanished after full static recrystallization (SRX) because of encroachment by the preferentially nucleated $\{101\}$ grains at hear bands. Therefore, the extraordinary texture in the $\mathrm{HN}$ was completely replaced to a common one after SRX. Dense SRX nucleation in the HNed SUS316LN led to uniform evolution of fine grains as fine as a few micrometers in average. Well-balanced tensile properties of yield and ultimate tensile strength of about $642 \mathrm{MPa}$ and $940 \mathrm{MP}$ at best were achieved without spoiling ductility. The tensile strength tended to increase with increasing Mn concentration, while the microstructure appeared not to be changed. To suppress $\{101\}$ texture evolution after SRX of the HNed SUS316LN, preferential nucleation at shear bands must be overwhelmed.

\section{Acknowledgements}

Some of the authors, H. Miura, M. Kobayashi, appreciate the financial support given by Japan Science and Technology Agency (JST) under Industry- Academia Collaborative R\&D Program "Heterogeneous Structure Control: Towards Innovative Development of Metallic Structural Materials" (Grant \#: JPMJSK1413).

\section{REFERENCES}

1) H. Inoue and T. Takasugi: Mater. Trans. 48 (2007) 2014-2022.

2) T. Sakai, Y. Saito and K. Kato: Trans. ISIJ 27 (1987) 520-525.

3) S. Umehara, H. Inoue and J. Hamada: J. Japan Inst. Met. Mater. 81 (2017) 542-546.

4) H. Miura, M. Kobayashi, Y. Todaka, C. Watanabe and Y. Aoyagi: J. Japan Inst. Met. Mater. 81 (2017) 536-541.

5) C. Donadille, R. Valle, P. Dervin and R. Penelle: Acta Metall. 37 (1989) 1547-1571.

6) H. Minami, Y. Funakawa, T. Tsuji, M. Kobayashi and H. Miura: ISIJ Int. 61 (2021) 2159-2166.

7) H. Miura, M. Kobayashi, Y. Todaka, C. Watanabe, Y. Aoyagi, N. Sugiura and N. Yoshinaga: Scr. Mater. 133 (2017) 33-36.

8) F. Khodabakhshi, M. Mohammadi and A.P. Gerlich: J. Mater. Sci. 56 (2021) 15513-15537.

9) H. Miura, M. Kobayashi, C. Watanabe, N. Suigiura and N. Yoshinaga: Mater. Trans. 61 (2020) 416-419.

10) N. Narita and J.I. Takamura: Dislocation in Solids, Vol. 9, ed. by F.R.N. Nabarro, (Elsevier Science Publishers, 1992) Chapter 46, pp. $135-189$.

11) R.E. Schramm and R.P. Reed: Metall. Trans. A 6 (1975) 1345-1351.

12) C. Watanabe, S. Kobayashi, Y. Aoyagi, Y. Todaka, M. Kobayashi, N. Sugiura, N. Yoshinaga and H. Miura: Tetsu-to-Hagané 105 (2019) 254-261.

13) N. Koga, S. Suzuki, H. Jiang, C. Watanabe, Y. Aoyagi, M. Kobayashi and H. Miura: J. Mater. Sci. 55 (2020) 9299-9310.

14) H. Miura, T. Sakai, R. Mogawa and J.J. Jonas: Philos. Mag. 87 (2007) 4197-4209. 